\title{
Giant persistent current in free-electron model with flat Fermi surface.
}

\author{
E. V. Tsiper, A. L. Efros \\ Department of Physics, University of Utah, Salt Lake City, Utah, 84112
}

(January 1, 2018)

\begin{abstract}
For the first time the persistent current in a 2D free-electron system has been calculated analytically. The tight binding model is considered on a square lattice with filling factor $1 / 2$. The array has a shape of rectangle with boundary conditions in both directions twisted by $2 \pi \phi_{x}$ and $2 \pi \phi_{y}$. The components of the twist are associated with two components of the magnetic flux in torus geometry. An analytical expression is obtained for the energy and for the components of the persistent current (PC) at a given flux and temperature. It is shown that at zero temperature the $\mathrm{PC}$ density is proportional to the vector potential with the coefficient which does not depend on the size of the system. This happens because the Fermi surface for a square lattice at filling factor $1 / 2$ is flat. Both the energy and the PC are periodic functions of the two flux components with the periods $\phi_{0} / q$ and $\phi_{0} / s$ where $\phi_{0}=h c / e$, and $q$ and $s$ are integers which depend on the aspect ratio of the rectangle. The magnitude of $\mathrm{PC}$ is the same as in superconductors. Therefore, a 3D system constructed from a macroscopic number of isolated coaxial cylinders at zero temperature reminds the London's superconductor. It exhibits the quantization of trapped flux as well as the Meissner effect. However, all the phenomena are of a mesoscopic nature. The critical field $H_{c}$ decays with an
\end{abstract}


effective size of the system, $H_{c} \sim 1 / R_{e f}$. The magnitude of PC decays with $T$ as $\exp \left(-\pi T R_{e f} / 2 a t\right)$, where $t$ is the hopping amplitude and $a$ is the lattice constant.

73.23.-b, 04.20.Jb, 71.45.-d, 75.20.-g

Typeset using REVTEX 


\section{INTRODUCTION}

Persistent current (PC) in mesoscopic structures1 1 月 has been extensively studied during the last decade both experimentally 3 目 and theoretically. The theoretical investigations concentrated on a role of different degrees of disorder 6 t and on the role of the interaction between electrons 8 .

The PC is a reaction of a system to an applied flux $\Phi$, or, equivalently, it can be described as a change of the energy of the system due to twisted boundary conditions. In a two-dimensional system which forms a cylinder the twisted conditions mean that the wave function of a system acquires a factor $\exp \left(i 2 \pi \Phi / \phi_{0}\right)$ with a circulation of one electron around the axis of the cylinder. Here $\phi_{0}=h c / e$ is the flux quanta.

The flux $\Phi$ is related to the tangential component $A$ of the vector potential $\Phi=2 \pi R A$, where $R$ is the radius of the cylinder. For a system with Galilean invariance the following simple statement is correct. The energy of a state with a given value of tangential component $P$ of the total momentum depends on $A$ as

$$
E(P, A)=E_{0}-\frac{1}{2 M}\left(P-\frac{N e}{c} A\right)^{2},
$$

where $N$ is the number of electrons and $M=N m$ is their total mass, $m$ being the mass of one electron. The $2 \mathrm{D}$ current density $j$ for a state with fixed $P$ is

$$
j_{P}=-\frac{c}{S}\left(\frac{\partial E}{\partial A}\right)_{P}=-\frac{n e^{2}}{m c} A+\frac{e P}{m S},
$$

where $S$ is the area of the cylinder surface and $n=N / S$. At $P=0$ Eq. (2) reminds the London equation for a superconducting current. In this case $n$ should be the superfluid density.

A general derivation of Eq. (2), given above, is misleading because PC should be defined as a current in the ground state rather than in a state with fixed $P$. In two- or threedimensional systems of free electrons the derivative of the energy with respect to $A$ cannot be taken in such a simple way because the intervals of $\Phi$, where branches of spectrum with different $P$ change each other in the ground state, tend to zero with increasing system size. 
For electrons in a periodic potential the situation is typically similar. The derivative of energy with respect to the flux is large for a given branch. However, different branches replace each other in the ground state at such small intervals of $\Phi$ that the derivative taken at a given total quasimomentum $P$ does not reflect properties of the ground state. Scalapino et al 11 considered a tight binding model on a two-dimensional square lattice. Their computations show that at filling factor $\nu=1 / 4$ the first level crossing occurs at $\Phi \sim 1 / L$, where $L$ is the size of the system. Their general conclusion is that the superfluid density, as found from the relation between $j$ and $A$, is zero for free electrons in the tight binding model. We show in this paper that this is not always the case.

Namely, we consider a 2D system of free electrons on a square lattice in a tight binding approximation at filling factor $\nu=1 / 2$. The shape of the system is assumed to be a rectangle with arbitrary aspect ratio. We demonstrate below that at $T=0$ the two-dimensional PC density does not depend on the size of the system and has a form:

$$
j=-\frac{4}{\pi^{2}} \frac{n e^{2}}{m c}\left(A-A_{0}\right) .
$$

Here $m=\hbar^{2} / 2 t a^{2}$ is the electron mass, $t$ being the nearest-neighbor hopping energy. The two-dimensional density is determined as $n=1 / 2 a^{2}$, where $a$ is the lattice constant. For simplicity, we consider a system of spinless fermions. The generalization to the case of non-interacting fermions with spin is straightforward.

The constant $A_{0}$ shows that the minimum of energy occurs not at zero flux. In contrast to Eq. (2), Eq. (3) describes PC in the ground state of the system which is a periodic function of $\Phi$ with period $\phi_{0} / q$. Eq. (3) is valid within the interval $0<\Phi<\phi_{0} / q$, or $0<A<\phi_{0} / 2 \pi R q$, and is to be repeated periodically for other values of flux. Here $q$ is an integer which depends on the aspect ratio of the cylinder and on the type of the boundary conditions imposed in the direction of the cylinder axis. The first term is shown to be independent on the aspect ratio.

We found PC to have an order of magnitude of the London current. Note that this result gives substantially larger $\mathrm{PC}$ than it is prescribed in the ballistic regime by the so-called 
$M$-channel approximation (see Ref. 10 and references therein). Namely, our exact solution gives PC larger by a factor of $\sqrt{L}$ for the $L \times L$ square. This discrepancy is due to the fact that in the case of flat Fermi surface all transverse channels are coherent.

Considering the 3D system constructed of a large number of coaxial closely packed 2D cylinders we show that it mimics the Meissner effect and the quantization of flux trapped in the opening.

These properties appear since the Fermi surface at $\nu=1 / 2$ is flat and no branch crossings occur in large intervals of $\Phi$. Say, for a square array no branch crossing occurs in the whole interval $0<\Phi<\phi_{0}$, which means that $q=1$.

In fact, we are discussing a mesoscopic effect. The expression Eq. (3) is valid only at mesoscopically small temperature, and the ideal diamagnetism occurs for mesoscopically small values of magnetic field:

$$
\begin{aligned}
& T<T_{c} \sim \frac{a}{R_{e f}} t \\
& H<H_{c} \sim \frac{a}{R_{e f}} \sqrt{\frac{t}{a^{2} b}}
\end{aligned}
$$

where $b$ is the spacing between neighboring coaxial cylinders. The effective size $R_{e f}$ is given by

$$
R_{e f}=s D=q 2 \pi R=\sqrt{s q 2 \pi R D}
$$

where $D$ is the length of the cylinders and $s$ and $q$ are integers determined by the aspect ratio $2 \pi R / D$ (see below). Thus, this system can be classified as a "mesoscopic superconductor."

Note that the average distance between energy levels in a $2 \mathrm{D}$ system is proportional to $1 / R^{2}$. The $1 / R$ behavior in the above equations is also a result of the flat Fermi surface at $\nu=1 / 2$. As it is seen from the calculations, all relevant interlevel distances are of the order of $(a / R) t$, rather than $(a / R)^{2} t$.

Since both $T_{c}$ and $H_{c}$ vanish at large $R$, there are no real critical phenomena in this model system. 


\section{CALCULATION OF PC AT ZERO TEMPERATURE}

Consider a rectangle of $L_{x} \times L_{y}$ lattice sites with periodic boundary conditions twisted in both directions by $2 \pi \Phi_{x} / \phi_{0}$ and $2 \pi \Phi_{y} / \phi_{0}$. This corresponds to a toroidal geometry where $\Phi_{x}$ is the flux through the crossection of the torus and $\Phi_{y}$ is the flux through the opening.

The single-electron energies have the form

$$
\epsilon\left(n_{x}, n_{y}, \phi_{x}, \phi_{y}\right)=-2 J\left\{\cos \left[\frac{2 \pi}{L_{x}}\left(n_{x}-\phi_{x}\right)\right]+\cos \left[\frac{2 \pi}{L_{y}}\left(n_{y}-\phi_{y}\right)\right]\right\}
$$

where we introduce dimensionless $\phi_{x, y}=\Phi_{x, y} / \phi_{0}$ to simplify the notation. The values of integer quantum numbers $n_{x}$ and $n_{y}$ are restricted to the rectangle $\left|n_{x, y}\right| \leq L_{x, y} / 2$ (first Brillouin zone). To find the energy of the ground state one has to sum $\epsilon\left(n_{x}, n_{y}\right)$ over the values $\left\{\left(n_{x}, n_{y}\right)\right\}$ inside the Fermi surface.

In principle, the calculation of PC can be performed either at constant number of particles $N$ or at constant value of chemical potential $\mu$. Generally speaking, these two definitions are not equivalent. It is important to note that such a problem does not exist at $\nu=1 / 2$ at even numbers of $L_{x}, L_{y}$ at least. As one can see from the Eq. (7), every single-electron energy changes sign under the transformation $n_{x}, n_{y} \rightarrow n_{x}+L_{x} / 2, n_{y}+L_{y} / 2$. It follows, that at $\nu=1 / 2$ due to the electron-hole symmetry the chemical potential $\mu$ is zero at any value of flux and at any temperature. Thus, if the flux changes at $\mu=0$, the number of particles in the ground state of the system does not change and if the flux changes at a given number of particles such that $\nu=1 / 2$, the chemical potential does not change.

Let us define the Fermi "surface" (FS) in two-dimensional $n_{x}, n_{y}$ space by the equation

$$
\epsilon\left(n_{x}, n_{y}, \phi_{x}, \phi_{y}\right)=0
$$

considering $n_{x}, n_{y}$ as continuous variables. It is easy to see that the FS forms a rhomb at any value of flux. Change in the flux produces a shift of the FS as a whole without changing its shape.

First, let us consider for simplicity a square sample, $L_{x}=L_{y}$. The FS forms a square shown in Fig. 1(a). At $\phi_{x, y}=0$ some of the allowed single-electron states lie exactly at the 
sides of this square. All the states inside the square and $1 / 2$ of the states at the sides of the square are occupied. All the states at the sides have the same energy so the occupation numbers of these states are not defined, while the many-electron ground state is degenerate.

The degeneracy is lifted at infinitazimally small values of $\phi$. Suppose that $\phi_{y}=0$ and $\phi_{x}>0$. Then FS is shifted to the right (See Fig. 1(a)). All occupation numbers become defined. Namely, the states at the right side of initial square get occupied and those at the left side become empty. Note that the occupation numbers of as many as $2 L$ states change when $\phi_{x}$ crosses zero.

It is easy to see that the occupation numbers are constant throughout the interval $0<$ $\phi_{x}<1$. The total energy decreases with $\phi_{x}$ and then increases again. At $\phi_{x}=1$ all electrons jump one step to the right and the Fermi surface restores its original position with respect to the lattice of integer numbers $\left(n_{x}, n_{y}\right)$. The total energy thus returns to the same value as at $\phi_{x}=0$.

It follows that the total quasimomentum of electron system in the ground state does not change through all this interval and no branch crossing occurs. Then the sum over the occupied states can be easily evaluated:

$$
\begin{aligned}
E\left(\phi_{x}, \phi_{y}\right) & =\sum_{n_{y}=1}^{L / 2-1} \sum_{n_{x}=-L / 2+n_{y}+1}^{L / 2-n_{y}}\left[\epsilon\left(n_{x}, n_{y}\right)+\epsilon\left(n_{x},-n_{y}\right)\right]+\sum_{n_{x}=-L / 2+1}^{L / 2} \epsilon\left(n_{x}, 0\right) \\
& =8 t \operatorname{Re} \frac{e^{2 \pi i / L}}{\left(e^{2 \pi i / L}-1\right)^{2}}\left[\left(1+e^{2 \pi i / L}\right) e^{-i 2 \pi \phi_{x} / L}+2 e^{-i 2 \pi \phi_{y} / L}\right]
\end{aligned}
$$

This expression is exact in the region $0<\phi_{x} \pm \phi_{y}<1$. In the limit of large $L$, the $\phi$ dependent part of the energy, $\delta E\left(\phi_{x}, \phi_{y}\right)=E\left(\phi_{x}, \phi_{y}\right)-E(0,0)$, can be written in the form:

$$
\delta E\left(\phi_{x}, \phi_{y}\right)=8 t\left[\phi_{y}^{2}-\phi_{x}\left(1-\phi_{x}\right)\right]
$$

Repeating Eq. (10) periodically one gets the expression valid in the whole plane $\left(\phi_{x}, \phi_{y}\right)$ :

$$
\delta E\left(\phi_{x}, \phi_{y}\right)=4 t\left[\left(\left\{\phi_{+}\right\}-\frac{1}{2}\right)^{2}+\left(\left\{\phi_{-}\right\}-\frac{1}{2}\right)^{2}-\frac{1}{2}\right],
$$

where $\phi_{ \pm}=\phi_{x} \pm \phi_{y}$, and $\{\ldots\}$ denotes the fractional part of the number, defined as a difference between the number and the largest integer less than it. 
Fig. 2 shows the energy $\delta E\left(\phi_{x}, \phi_{y}\right)$ as given by Eq. (11). The positions of energy minima form a square lattice shifted from the origin:

$$
\left(\phi_{x}, \phi_{y}\right)=\left(\frac{1+i+j}{2}, \frac{i-j}{2}\right)
$$

with arbitrary integer $i$ and $j$.

The point $\phi_{x}=\phi_{y}=0$ corresponds to a maximum of energy, in the same way as in $1 \mathrm{D}$ case with even number of electrons. At this point the derivatives $d \delta E / d \phi_{x, y}$ are discontinuous. Such behavior appears as a result of lifting of the degeneracy of the states at the Fermi surface.

The PC at $T=0$ can be found as the derivative of the total energy with respect to flux:

$$
\begin{aligned}
I_{x, y} & =-c \frac{\partial E}{\partial \Phi_{x, y}}=-\frac{c}{\phi_{0}}\left(\frac{\partial E}{\partial \phi_{+}} \pm \frac{\partial E}{\partial \phi_{-}}\right) \\
& =-8 \frac{c t}{\phi_{0}}\left[\left(\left\{\phi_{+}\right\}-\frac{1}{2}\right) \pm\left(\left\{\phi_{-}\right\}-\frac{1}{2}\right)\right]
\end{aligned}
$$

The magnitude of $\delta E\left(\phi_{x}, \phi_{y}\right)$ and $I_{x, y}\left(\phi_{x}, \phi_{y}\right)$ as given by Eqs. (11), (13) is independent on the size $L$ of the square. Such a large magnitude results from the fact that in the region with no branch crossings (or, with no electron changing its state) all electrons together contribute to the current.

It may seem that the aspect ratio $L_{x} / L_{y}=1$ is crucial for the effect. In the next section we calculate $\mathrm{PC}$ at finite temperature for arbitrary aspect ratio $L_{x}=s K, L_{y}=q K$ with mutually-prime integers $s$ and $q$. We assume macroscopic limit $K \rightarrow \infty$. It is useful to generalize $\phi_{ \pm}$for a rectangular sample as

$$
\phi_{ \pm}=q \phi_{x} \pm s \phi_{y}
$$

In the limit $T=0$ we find

$$
\begin{aligned}
& I_{x}=-\frac{8}{s q} \frac{c t}{\phi_{0} / q}\left[\left(\left\{\phi_{+}\right\}-\frac{1}{2}\right)+\left(\left\{\phi_{-}\right\}-\frac{1}{2}\right)\right] \\
& I_{y}=-\frac{8}{s q} \frac{c t}{\phi_{0} / s}\left[\left(\left\{\phi_{+}\right\}-\frac{1}{2}\right)-\left(\left\{\phi_{-}\right\}-\frac{1}{2}\right)\right]
\end{aligned}
$$


The flux-dependent part of the energy can be restored from Eq. (15):

$$
\delta E\left(\phi_{x}, \phi_{y}\right)=\frac{4 t}{s q}\left[\left(\left\{\phi_{+}\right\}-\frac{1}{2}\right)^{2}+\left(\left\{\phi_{-}\right\}-\frac{1}{2}\right)^{2}-\frac{1}{2}\right],
$$

This result is a generalization of Eq. (11) to an arbitrary aspect ratio $s / q$ of the rectangular sample.

As follows from Eqs. (15), (16), the energy and current as functions of flux do depend on the aspect ratio. However, they do not depend on the system size, if the aspect ratio is kept constant.

The result Eqs. (15), (16), can be understood from Fig. 1(b), which is drawn for the case $L_{x} / L_{y}=2 / 3$. Contrary to the Fig. $1\left(\right.$ a), there are now points $\left(n_{x}, n_{y}\right)$ closer to the Fermi surface than one lattice spacing. However, there is still a regularity in their positions. Namely, as the Fermi surface shifts with flux, the points enter the Fermi sea in groups. Consider, for example, the same case as above: $\phi_{y}=0$ and $\phi_{x}>0$. As seen in Fig. 1(b), the branch crossings occur only at $\phi_{x}=2 \pi / 3,4 \pi / 3$, and $2 \pi$. In terms of $\phi_{ \pm}$this corresponds to integer $\phi_{ \pm}=3 \phi_{x}=1,2$, and 3 . These values of flux are determined by $s$ and $q$ and do not change with the size of the system. The number of points in each group, in tern, is proportional to the size of the system, so the corresponding contribution to the current is large.

At $\Phi_{y}=0$ Eq. (15) gives

$$
I_{x}=-\frac{16}{s q} \frac{c t}{\phi_{0} / q}\left(\left\{\frac{\Phi_{x}}{\phi_{0} / q}\right\}-\frac{1}{2}\right) .
$$

Up to now we are discussing the torus geometry. To come to a cylinder geometry one has to formulate the boundary conditions in the direction of the cylinder axis, chosen as $y$. In what follows we assume periodic boundary conditions in this direction with $\Phi_{y}=0$. This leads to Eq. (17) for a total current through the cylinder. As another option me may impose the condition that the wave function is zero at the edges of the cylinder. It can be shown that in this case the second term in Eq. (17) changes while the first term remains intact.

Note that both energy and current are periodic functions of flux with period $\phi_{0} / q$ rather than $\phi_{0}$. 
Taking into account that the current density $j_{x}=I_{x} /\left(a L_{y}\right)$ and that the vector potential $A_{x}=\Phi_{x} /\left(a L_{x}\right)$ one obtains Eq. (3) with the first term independent of $s$ and $q$.

\section{PC AT FINITE TEMPERATURE}

We start with the equation

$$
I_{x}=-\frac{c}{\phi_{0}} \sum_{n_{x}=0}^{s K-1} \sum_{n_{y}=0}^{q K-1} \frac{\partial \epsilon\left(n_{x}, n_{y}\right)}{\partial \phi_{x}} \frac{1}{1+\exp \left(\epsilon\left(n_{x}, n_{y}\right) / T\right)} .
$$

It is convenient to rewrite the single electron energy in the form

$$
\epsilon\left(n_{x}, n_{y}\right)=-4 t \cos \left(\frac{\pi}{s q K}\left(n_{+}-\phi_{+}\right)\right) \cos \left(\frac{\pi}{s q K}\left(n_{-}-\phi_{-}\right)\right)
$$

where $n_{ \pm}=q n_{x} \pm s n_{y}$ and $\phi_{ \pm}$are given by Eq. (14). Using $\partial / \partial \phi_{x}=q\left(\partial / \partial \phi_{+}+\partial / \partial \phi_{-}\right)$we find that the current has two terms,

$$
I_{x}=\frac{1}{s}\left(I_{+}+I_{-}\right)
$$

where

$$
I_{ \pm}=-s q \frac{c}{\phi_{0}} \sum_{n_{x}=0}^{s K-1} \sum_{n_{y}=0}^{q K-1} \frac{\partial \epsilon\left(n_{x}, n_{y}\right)}{\partial \phi_{ \pm}} \frac{1}{1+\exp \left(\epsilon\left(n_{x}, n_{y}\right) / T\right)}
$$

The idea of our calculation is to transform Eq. (21) in such a way that the internal sum gives $\mathrm{PC}$ of $1 \mathrm{D}$ problem with effective temperature and effective flux. For this purpose we use the identity:

$$
\sum_{n_{x}=0}^{s K-1} \sum_{n_{y}=0}^{q K-1} f\left(n_{x}, n_{y}\right)=\sum_{m=0}^{s K-1} \sum_{d=0}^{q-1} \sum_{k=0}^{K-1} f(m+d+s k, d+q k) .
$$

This identity is valid for any function $f\left(n_{x}, n_{y}\right)$ periodic in $n_{x}$ and $n_{y}$ with periods $s K$ and $q K$ respectively. Then $I_{+}$can be written in the form

$$
I_{+}=-\frac{4 \pi c t}{K \phi_{0}} \sum_{m=0}^{s K} \sum_{d=0} \sum_{k=0}^{q-1} \frac{\sin \left(\frac{\pi}{s q K}\left(n_{+}-\phi_{+}\right)\right) \cos \left(\frac{\pi}{s q K}\left(n_{-}-\phi_{-}\right)\right)}{1+\exp \left[-\frac{4 t}{T} \cos \left(\frac{\pi}{s q K}\left(n_{+}-\phi_{+}\right)\right) \cos \left(\frac{\pi}{s q K}\left(n_{-}-\phi_{-}\right)\right)\right]} .
$$

Similar expression can be written for $I_{-}$. Note that $n_{-}=q m+(q-s) d$ does not depend on $k$, while $n_{+}=(q m+q d+s d)+2 s q k$ does depend on $k$. Therefore, the current $I_{+}$can be written as 


$$
I_{+}=\sum_{m=0}^{s K-1} \sum_{d=0}^{q-1} \mathcal{I}_{+}(m, d)
$$

where $\mathcal{I}_{+}(m, d)$ denote the internal sum over $k$,

$$
\mathcal{I}_{+}(m, d)=-\frac{4 \pi c t}{K \phi_{0}} \frac{2 T}{\widetilde{T}} \sum_{k=0}^{K-1} \frac{\sin \left(\frac{2 \pi}{K}\left(k-\widetilde{\phi}_{+}\right)\right)}{1+\exp \left[-\frac{2 t}{T} \cos \left(\frac{2 \pi}{K}\left(k-\widetilde{\phi}_{+}\right)\right)\right]} .
$$

The sum in Eq. (25) describes the PC in 1D system with effective temperature and effective flux given by

$$
\begin{aligned}
\widetilde{T}(m, d) & =\frac{T}{2 \cos \left(\frac{\pi}{s q K}\left(q m+(q-s) d-\phi_{-}\right)\right)} \\
\widetilde{\phi}_{+}(m, d) & =\frac{\phi_{+}-q m-q d-s d}{2 s q}
\end{aligned}
$$

Using the Poisson summation formula (see Ref. 15), one obtains

$$
\mathcal{I}_{+}(m, d)=\frac{8 \pi c T}{\phi_{0}} \sum_{l=1}^{\infty} \frac{\cos (l \pi K / 2)}{\sinh (l \pi \widetilde{T} K / 2 t)} \sin \left(2 \pi l \widetilde{\phi}_{+}\right)
$$

Performing the summation over $m$ and $d$ in Eq. (24) we note that $\widetilde{T}$ is a smooth function of $m / K$ and $d / K$. However, $\sin \left(2 \pi l \widetilde{\phi}_{+}\right)$has an oscillatory behavior for some $l$, so that the contribution of the corresponding harmonics vanishes in the limit $K \rightarrow \infty$. The oscillatory behavior is absent if $l$ is an integer multiple of $2 s q$. For these $l$, the sum over $m$ can be transformed into integral via $p=(\pi / s K) m$, while the sum over $d$ simply gives a factor $q$. Thus, one obtains

$$
I_{ \pm}=\sum_{l=1}^{\infty} A_{l} \sin \left(2 \pi l \phi_{ \pm}\right)
$$

where

$$
A_{l}=s q K T \frac{2 c}{\phi_{0}} \int_{0}^{\pi} \frac{d p}{\sinh (l \pi s q K T / 2 t \sin p)}
$$

For the PC in $x$-direction one has from Eq. (20)

$$
I_{x}=\frac{1}{s} \sum_{l=1}^{\infty} A_{l}\left[\sin \left(2 \pi l \phi_{+}\right)+\sin \left(2 \pi l \phi_{-}\right)\right]
$$


Similar calculation gives

$$
I_{y}=\frac{1}{q} \sum_{l=1}^{\infty} A_{l}\left[\sin \left(2 \pi l \phi_{+}\right)-\sin \left(2 \pi l \phi_{-}\right)\right] .
$$

Eqs. (29), (30) give the Fourier series expansion of PC at any temperature. Expansion of $A_{l}$ at small $K T / t$ yelds

$$
A_{l} \approx \frac{8 t}{\phi_{0}} \frac{1}{l \pi}
$$

In this case Eqs. (30), (31), and (32) give the Fourier series expansion of the zero-temperature result Eq. (15).

In the opposite limit, $K T / t \gg 1$, the amplitudes of the harmonics decay as

$$
A_{l} \approx \frac{8 c}{\phi_{0} \sqrt{l}} \sqrt{s q K T t} \exp \left(-\frac{l \pi s q K T}{2 t}\right)
$$

so that $\mathrm{PC}$ is dominated by its lowest harmonic. When $\Phi_{y}=0$ one has

$$
I_{x} \approx \frac{1}{s q} \frac{16 c}{\phi_{0} / q}\left(\frac{R_{e f} T t}{a}\right)^{1 / 2} \exp \left(-\frac{\pi R_{e f} T}{2 a t}\right) \sin \left(\frac{\Phi_{x}}{\phi_{0} / q}\right) .
$$

\section{LOW TEMPERATURE MAGNETIC PROPERTIES}

In this section we study magnetic properties of a quasi-3D system constructed of a macroscopic number of closely packed coaxial cylinders assuming that the temperature is very low. Then the connection between flux and current for each cylinder is given by Eq. (17). For the sake of simplicity we assume that the cylinders are long, such that the circumference of the internal cylinder $2 \pi R=a L_{x}$ is much larger than $D=a L_{y}$. The distance between the internal and external cylinders is supposed to be much less than $R$. We assume further that all the cylinders have the same ratio $L_{x} / L_{y}=s / q$. One can imagine a small change either in $L_{x}$ and $L_{y}$ of adjacent cylinders or in their lattice constant.

The second term in Eq. (17) appears since zero flux does not correspond to the minimum

of energy. It may lead to an appearance of a spontaneous flux in this system. This idea has been put forward by Wohlleben et al, and Szopa and Zipper, Ref. 12, and then studied in 
details in Ref. 13. These authors considered a cylinder constructed from isolated 1D rings. Loss and Martin14 argued that in a single 1D ring no symmetry breaking can occur, but their arguments are restricted to 1D case.

In this paper we concentrate on the first term in Eq. (17). It is an analog of the London current in superconductors and it creates a strong diamagnetism in a quasi-3D system described above. Suppose that an external magnetic field $H_{\text {ext }}$ is applied to the system and that there is a solenoid creating flux $\Phi_{\text {ext }}$ inside the internal cylinder.

Let $\Phi_{k}$ be the total flux inside cylinder $k$, where $k=1$ for the internal cylinder and $k=N$ for the external one. The flux obeys the equation

$$
\Phi_{k}-\Phi_{k-1}=2 \pi R b\left(H_{e x t}+\frac{4 \pi}{c D} \sum_{i=k}^{N} I\left(\Phi_{i}\right)\right) .
$$

Here $b$ is the distance between adjacent cylinders which we assume to be of the order of the lattice constant $a$. Since the thickness $d=N b$ is supposed to be much less than $R$ we have neglected that the radii of cylinders are slightly different. The right hand side of Eq. (35) describes the flux through the area between the $k$-th and $(k-1)$-th cylinders created by the external field and outer cylinders.

The following condition should be added to this finite difference equation:

$$
\Phi_{1}-\Phi_{e x t}=\pi R^{2}\left(H_{e x t}+\frac{4 \pi}{c D} \sum_{i=1}^{N} I\left(\Phi_{i}\right)\right) .
$$

If $\Phi_{k}$ is a smooth function of $k$ one can transform Eq. (35) into differential equation

$$
\frac{d^{2} \Phi}{d r^{2}}=\frac{\phi_{0} / q}{\lambda^{2}}\left(\left\{\frac{\Phi}{\phi_{0} / q}\right\}-\frac{1}{2}\right) .
$$

Here $\lambda$ is the analog of the London penetration depth

$$
\lambda^{-2}=\frac{4 \pi}{b} \frac{16 t}{\phi_{0}^{2}}=\frac{16 e^{2} n_{3}}{\pi m c^{2}},
$$

where $n_{3}=1 / 2 b a^{2}$ is the $3 \mathrm{D}$ electron density.

Eq. (36) transforms into the boundary condition at $r=R$ :

$$
\Phi(R)-\Phi_{e x t}=\left.\frac{R}{2} \frac{d \Phi}{d r}\right|_{r=R} .
$$


The second boundary condition reads

$$
\left.\frac{d \Phi}{d r}\right|_{r=R+d}=2 \pi R H_{e x t}
$$

One can use differential equation if $\lambda \gg b$.

Eq. (37) can also be obtained by minimizing total energy with respect to flux. The total energy consists of two parts. First is the energy of magnetic field in the space between cylinders. The magnetic field can be expressed through $d \Phi / d r$ using Eq. (35) as

$$
\frac{d \Phi}{d r}=2 \pi R H(r)
$$

The second part is the internal energy of $2 \mathrm{D}$ electron gas. This energy per cylinder is given by Eq. (16) at $\phi_{y}=0$. Thus, one gets for the total energy

$$
E_{\text {total }}=\frac{1}{8 \pi} \frac{D}{2 \pi R} \int\left(\frac{d \Phi}{d r}\right)^{2} d r+\int \delta E(\Phi) \frac{d r}{b} .
$$

Minimizing this expression with respect to $\Phi(r)$ and taking into account that $d \delta E / d \Phi=$ $(-1 / c) I(\Phi)$, where $I(\Phi)$ is given by Eq. (17), one obtains Eq. (37).

The Eq. (37) is nonlinear since it contains the fractional part $\left\{\Phi /\left(\phi_{0} / q\right)\right\}$ which makes the right-hand side periodic. However, it becomes linear if the total drop of the flux inside the system is smaller than $\phi_{0} / q$. If $H_{e x t}=0$ the solution of the linearized equation with boundary conditions (39), (40) in the case $R \gg d \gg \lambda$ is

$$
\Phi(r)=\Phi_{n}+\left(\Phi_{e x t}-\Phi_{n}\right) \frac{2 \lambda}{R} \exp \left(-\frac{r-R}{\lambda}\right) .
$$

Here

$$
\Phi_{n}=\frac{\phi_{0}}{q}\left(n-\frac{1}{2}\right)
$$

One can see that the flux inside the cylinder with $d \gg \lambda$ may take only quantized values $\Phi_{n}$ with arbitrary integer $n$. Note that there is no zero flux among the allowed values of the frozen flux $\Phi_{n}$. This is because zero flux does not correspond to a minimum of the total energy at zero temperature. The solution Eq. (43) is obtained in the linear approximation 
and it is valid if $\left(\Phi_{\text {ext }}-\Phi_{n}\right) 2 \lambda / R<\phi_{0} / q$. The physics of this result is that the inner cylinders carry a current which creates a favorable flux for the rest of the system.

If the system is in an external magnetic field $H_{\text {ext }}$, the solution is

$$
\Phi(r)=\Phi_{n}+2 \pi R \lambda H_{e x t} \exp \left(-\frac{R+d-r}{\lambda}\right) .
$$

or in terms of magnetic field defined by Eq. (41)

$$
H(r)=H_{e x t} \exp \left(-\frac{R+d-r}{\lambda}\right)
$$

In this case the cylinders near external surface carry current which screens magnetic field inside the system and adjusts the total flux to $\Phi_{n}$. The solution is valid if $2 \pi R \lambda H_{e x t}<\phi_{0} / q$. This condition is equivalent to Eq. (5). It has a simple interpretation. The loss in the total energy due to the ideal Meissner effect is of the order of $H_{e x t}^{2} R D b$ per cylinder. The gain in the energy of a cylinder due to the adjusted flux is of the order of $t / s q$ (see Eq. (16)). At large field the loss becomes larger than the gain and the field penetrates into the system. This is the origin of a "mesoscopic" critical field. Note that the relation $H_{c}^{2} R D b \sim t / s q$ is also equivalent to Eq. (5).

It follows from the results of the previous section that zero-temperature approximation is good if $s q K T / t=T \sqrt{s q 2 \pi R D} /$ at $\ll 1$. This is the same condition as Eq. (田). At larger temperatures the penetration depth $\lambda$ increases as $\exp \left(\pi T R_{e f} / 4 a t\right)$ and eventually reaches the thickness $d$ of the cylinder, gradually destroying strong diamagnetism.

\section{CONCLUSIONS}

Finally we have presented a model which mimics in a mesoscopic scale some properties of superconductors, such as Meissner effect and quantization of flux, though the physics of the model does not involve any electron pairing. The flux quanta in the model is $\phi_{0} / q$ where $q$ is determined by the aspect ratio of the system.

Since the range of temperature and magnetic field for these phenomena shrinks to zero in a macroscopic system, one should not expect any phase transitions. However, for a 
mesoscopic system this range is not necessarily small. Let us assume a hypotetic 3D layered system with very weak interaction between layers and flat two-dimensional Fermi surface. Then it follows from Eqs. (4), (5) that the temperature range is up to $12 \mathrm{~K}$ and the range

of $H_{\text {ext }}$ is up to 240 gauss for a system with $R_{e f}=3 \times 10^{-5} \mathrm{~cm}, a=b=3 \times 10^{-8} \mathrm{~cm}$, and $t=1 \mathrm{eV}$. In a system with disorder the obvious condition for these phenomena is that the elastic mean free path is smaller than the size $R_{e f}$.

Our model ignores electron-electron interaction. We hope that it is not important at large $t$. Our modeling of small interacting systems up to 18 electrons show the same value of the PC at $t$ immediately above the Wigner crystal quantum melting point 1 .

\section{ACKNOWLEDGMENTS}

The authors thank D.E. Khmelnitskii for helpful discussions. The work was supported by QUEST of UCSB, subcontract KK3017. 


\section{FIGURES}

FIG. 1. The Fermi surface at $\nu=1 / 2$. The points represent allowed integer values of $n_{x}$ and $n_{y}$ inside the first Brillouin zone. The dashed lines show the Fermi surface at zero flux. The solid lines are the Fermi surface shifted by flux. The aspect ratio $L_{x} / L_{y}=1$ (a) and $L_{x} / L_{y}=2 / 3$ (b).

FIG. 2. Lines of constant $\delta E\left(\phi_{x}, \phi_{y}\right)$ as given by Eq. (16) for square sample $s=q=1$ (a), and for rectangle with $s=2$ and $q=3(\mathrm{~b})$. Note the difference in periodicities. 


\section{REFERENCES}

${ }^{1}$ W. Kohn, Phys. Rev. 133, A171 (1964).

${ }^{2}$ M. Buttiker, Y. Imry, and R. Landauer, Phys. Lett. 96, A365 (1983).

${ }^{3}$ L. P. Levy, G. Dolan, J. Dunsmuir, and H. Bouchiat, Phys. Rev. Lett. 64, 2074 (1990).

${ }^{4}$ V. Chandrasekhar, R. A. Webb, M. J. Brady, M. B. Ketchen, W. J. Gallagher, and A. Kleinsasser, Phys. Rev. Lett. 67, 3578 (1991).

${ }^{5}$ D. Mailly, C. Chapelier, A. Benoit, Phys. Rev. Lett. 70, 2020 (1993).

${ }^{6}$ A. Schmid, Phys. Rev. Lett. 66, 80 (1991); B. L. Altshuler, Y. Gefen, and Y. Imry, Phys. Rev. Lett. 66, 88 (1991).

${ }^{7}$ A. Altland, Y. Gefen, and G. Montambaux, Phys. Rev. Lett. 76, 1130 (1996).

${ }^{8}$ R. Berkovits, Y. Avishai, Solid State Commun. 93, 301 (1995).

${ }^{9}$ A. L. Efros, E. V. Tsiper, F. G. Pikus, Proc. of 23 Int. Conference on the Physics of Semiconductors, v. 4, p. 2439 (1996).

${ }^{10}$ H.-F. Cheung, E. K. Riedel, and Y. Gefen, Phys. Rev. Lett. 62, 587 (1989).

${ }^{11}$ D. J. Scalapino, S. R. White, and S. Zhang, Phys. Rev. B 47, 7995 (1993).

${ }^{12}$ D. Wohlleben, M. Esser, P. Freche, E. Zipper, and M. Szopa, Phys. Rev. Lett. 66, 3191 (1991); M. Szopa and E. Zipper, Solid State Comm. 77, 739 (1991).

${ }^{13}$ M. Szopa and E. Zipper, Int. J. of Modern Phys. 9, 161 (1995); M. Szopa, D. Wohlleben, and E. Zipper, Phys. Lett. A160, 271 (1991).

${ }^{14}$ D. Loss and T. Martin, Phys. Rev. B 47, 4619, 1993.

${ }^{15}$ H.-F. Cheung, Y. Gefen, E. K. Riedel, and W.-H. Shih, Phys. Rev. B 37, 6050 (1988).

16 to be published elsewhere. 


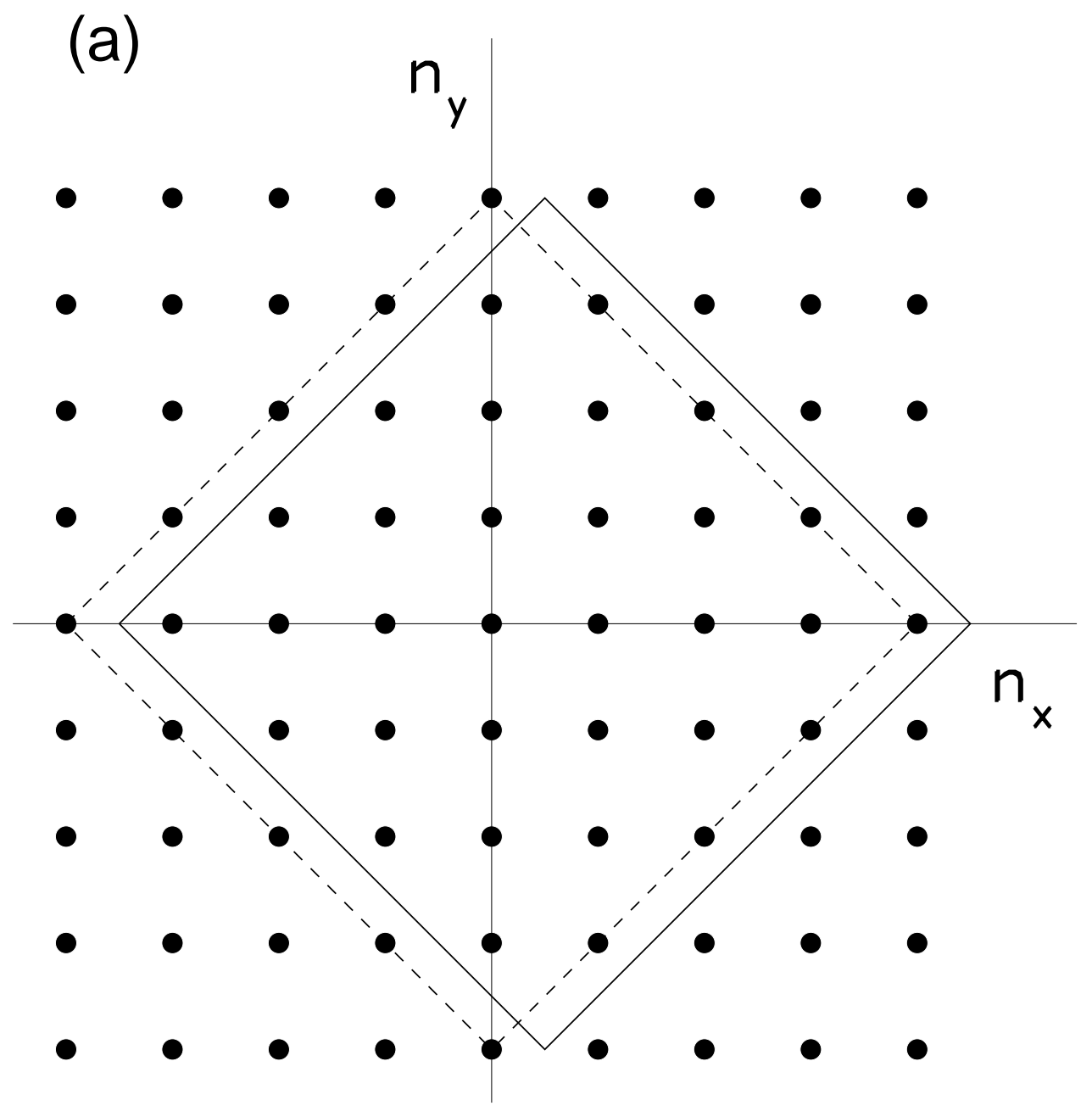




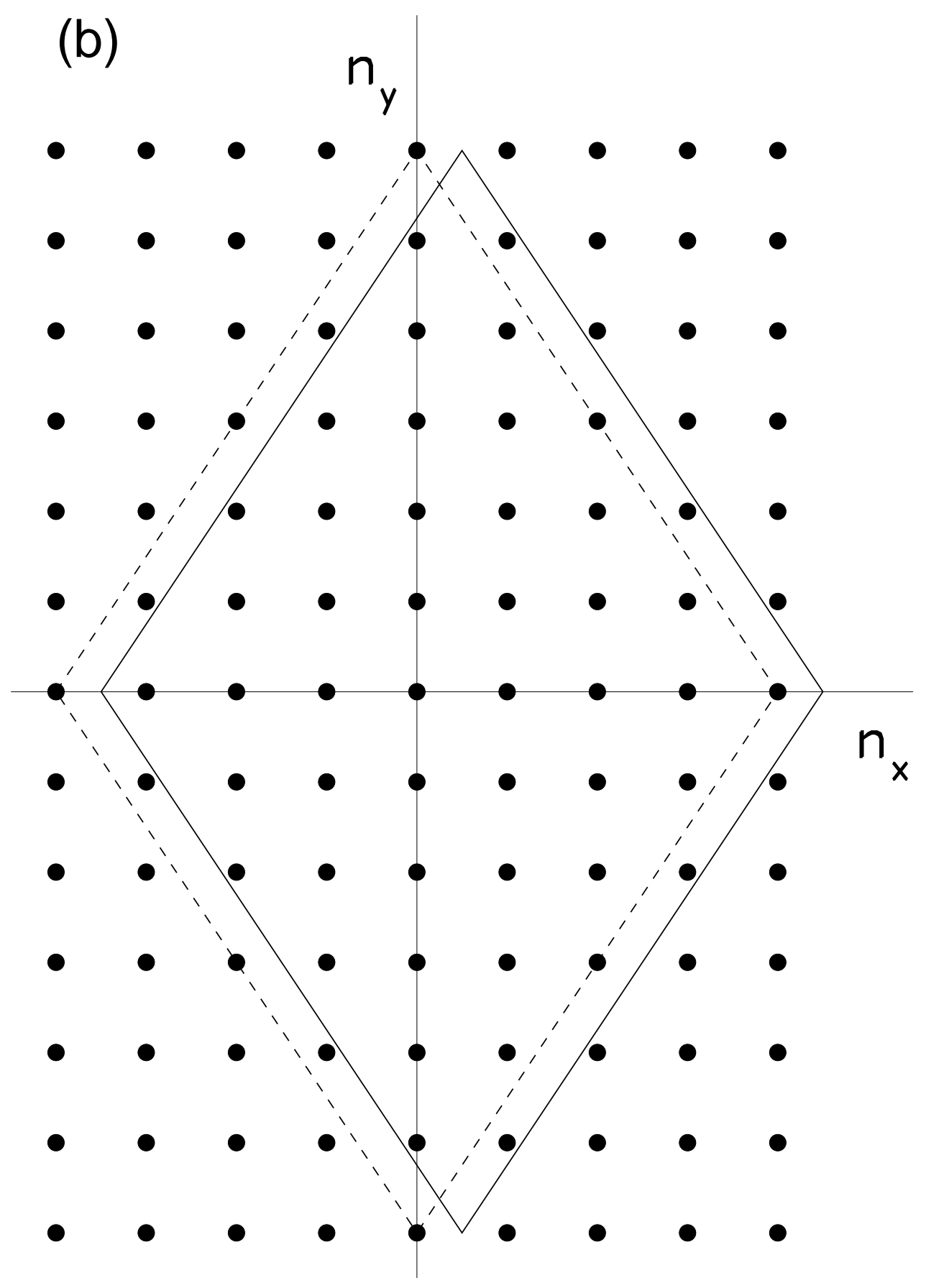




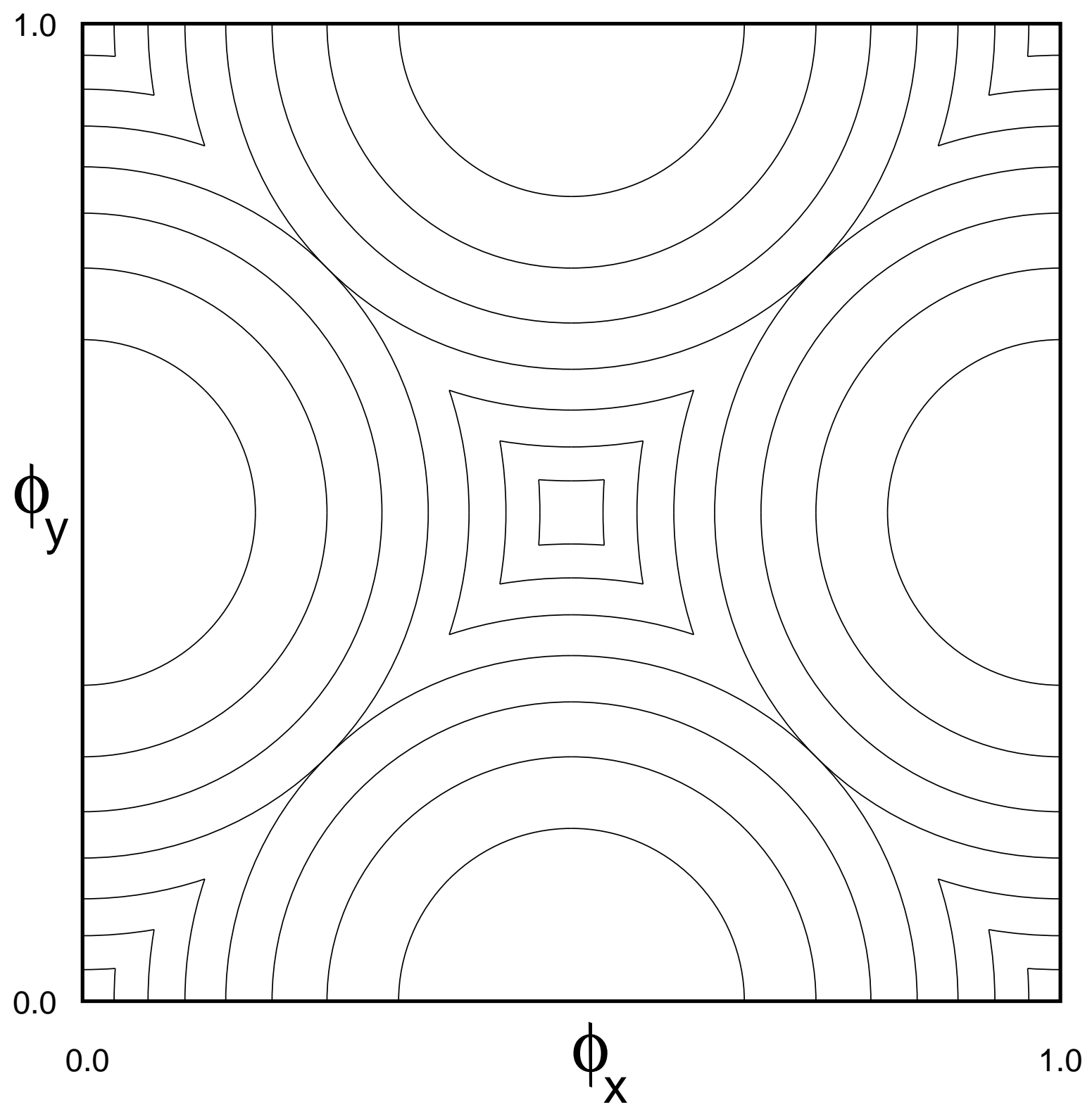

[E.V. Tsiper and A.L. Efros, Giant Persistent Current..., Fig. 2(a)] 


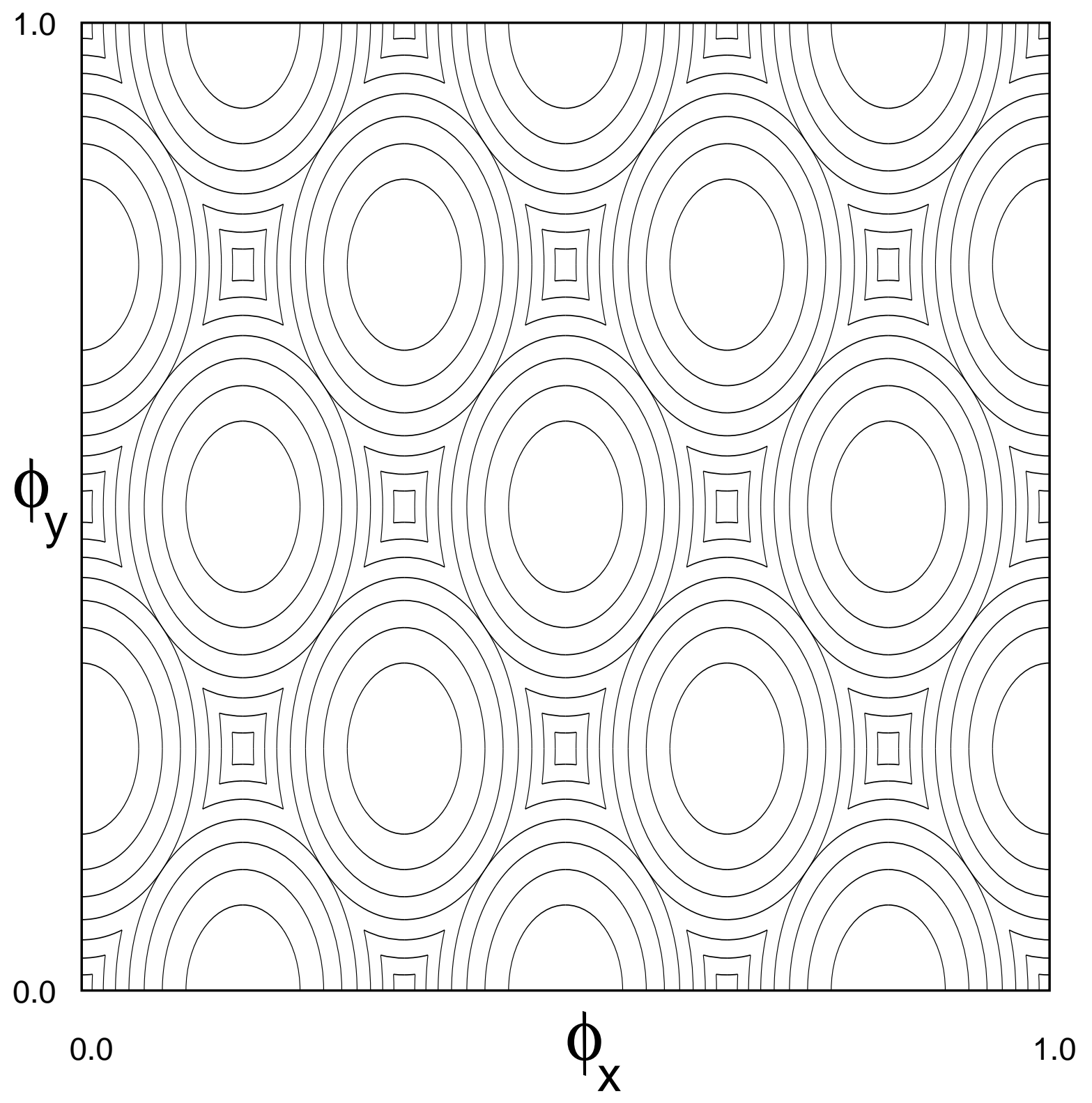

[E.V. Tsiper and A.L. Efros, Giant Persistent Current..., Fig. 2(b)] 